\title{
Monitoring Farmed Fish Welfare by Measurement of Cortisol as Stress Marker in Fish Feces by Liquid Chromatography Cou- pled with Tandem Mass Spectrometry
}

\author{
Vanessa A. Meling ${ }^{1}$, Kjetil Berge ${ }^{2}$, David L. Knudsen ${ }^{3}$, Per O. Rønning ${ }^{1}$ and Cato Brede ${ }^{3,4,5 *}$
}

1 Department of Mechanical, Electronic and Chemical Engineering, Faculty of Technology, Art and Design, Oslo Metropolitan University, N-0166 Oslo, Norway

2 Skretting AS, N-4016 Stavanger, Norway

3 Fishlab AS, N-4015 Stavanger, Norway

4 Department of Chemistry, Bioscience and Environmental Engineering, University of Stavanger, N-4036 Stavanger, Norway

5 Department of Medical Biochemistry, Stavanger University Hospital, N-4068 Stavanger, Norway

* Correspondence: cato.brede@uis.no

\begin{abstract}
:
The aquaculture industry has become a sustainable provider of food to humans. Remaining challenges include disease issues, as well as ethical concerns for discomfort and stress among farmed fish. There is a need for reliable biomarkers to monitor welfare in fish, and the stress hormone cortisol has been suggested as a good candidate. This study presents a novel method for measurement of cortisol in fish feces based on enzymatic hydrolysis, liquid-liquid extraction, derivatization, and finally instrumental analysis by liquid chromatography coupled with tandem mass spectrometry. Hydrolysis and extraction conditions were optimized. Cortisol appeared to be mostly conjugated to sulfate and less conjugated to glucuronic acid in the studied samples of feces from farmed Atlantic salmon. The method was suitable for quantification of cortisol after enzymatic deconjugation by either combined glucuronidase and sulfatase activity, or by glucuronidase activity alone. The limit of detection was $0.15 \mathrm{ng} / \mathrm{g}$ and the limit of quantification was $0.34 \mathrm{ng} / \mathrm{g}$, and the method was linear $\left(R^{2}>0.997\right)$ up to $380 \mathrm{ng} / \mathrm{g}$, for measurement of cortisol in wet feces. Method repeatability and intermediate precision were acceptable, both with a CV of $11 \%$. Stress level was high in fish released into seawater, and significantly reduced after eight days.
\end{abstract}

Keywords: aquaculture, fish, salmon, feces, deconjugation, hydrolysis, extraction, chromatography, mass spectrometry, LC-MS/MS

\section{Introduction}

In Norway, aquaculture has grown to become the second-largest export industry, after oil and gas [1]. The most common farmed fish is Atlantic salmon (Salmo salar). Despite of its success, the industry is still facing issues around fish health and welfare that need to be resolved [2]. Therefore, identification of early markers of stress can be of high value not only to prevent an outbreak of disease and economical losses but also to ensure proper ethical farming conditions.

The stress hormone cortisol is a useful marker of stress in fish [3] and it is well established that plasma cortisol rises quickly during stressful events. However, sampling of blood from fish can be such an event, leading to erroneous results. Measurement of cortisol in feces is therefore a better approach, as it is likely to correlate with stress experienced some time before the sampling is done. Furthermore, collection of feces from fish is a less invasive procedure [4]. Direct measurement of corticoid metabolites in fish feces has previously been achieved by using enzyme-linked immunosorbent assay (ELISA), and this 
type of assessment was then proposed as a good indicator of fish welfare [5]. It is well known that immunoassays, such as ELISA, can overestimate analyte concentrations, especially when there is cross-reactivity with metabolites. Analysis by high-performance liquid chromatography coupled with UV detection (HPLC-UV) has also been reported [6] but has the disadvantage of low sensitivity. Today, liquid chromatography coupled with tandem mass spectrometry (LC-MS/MS) is well-established in clinical laboratories for selective and sensitive measurement of cortisol and other steroid hormones at low concentration levels in biological samples [7]. This technique utilizes electrospray ionization (ESI) where methods often depend on derivatization to improve ionization efficiency [8]. For this purpose, we recently investigated several derivatization reagents suitable for improved detectability of steroid hormones in saliva [9]. In the present work, we present a novel method for measurement of cortisol in fish feces by liquid chromatography coupled with tandem mass spectrometry (LC-MS/MS). Before instrumental analysis, samples were deconjugated by beta-glucuronidase enzymes, either with or without sulfatase activity. Increased detectability of cortisol was achieved by liquid-liquid extraction and derivatization. Finally, we applied the new method for analysis of feces from farmed Atlantic salmon, allowing us to identify fish groups with elevated stress levels.

\section{Materials and Methods}

\subsection{Chemicals and Solutions}

Cortisol and isotopic labeled D4 cortisol (hydrocortisone-9,11,12,12-d4) used as internal standard were both from Merck KGaA (Darmstadt, Germany). LC-MS grade methanol and acetonitrile (ACN), and analytical grade 1-propanol, 1-butanol, methyl tert-butyl ether (MTBE), sodium chloride, hydrochloric acid, acetic acid, formic acid, and 25\% ammonium hydroxide solution were all from VWR International (Radnor, PA, USA). Water was type I purified to 18.2 MOhm (MilliporeSigma, Burlington, MA, United States). Aqueous solutions of beta-glucuronidase from Helix Pomatia (product number G1707) and Patella Vulgata (product number G2174) were from Merck. Beta-glucuronidase with sulfatase $\left(\mathrm{Abalonase}^{\mathrm{TM}}+\right.$ ) or without added sulfatase (Abalonase ${ }^{\mathrm{TM}}$ ) were from Ango (San Ramon, CA, USA). 4-Aminobenzoic hydrazide (4-ABH) was from TCI (Tokyo, Japan).

Stock and intermediate solutions of cortisol were made with methanol, while eight calibrator standard solutions in the $0-100 \mathrm{ng} / \mathrm{mL}$ range were made by dilution with water. Stock and user solution of D4 cortisol internal standard $(67 \mathrm{ng} / \mathrm{mL})$ were made with methanol. Saturated sodium chloride $(\mathrm{NaCl})$ solution (approximately $5 \mathrm{~mol} / \mathrm{L}$ ) was made by adding more salt than could be dissolved in water at room temperature. Ammonium acetate buffer $(2 \mathrm{~mol} / \mathrm{L})$ was made by pipetting $5 \mathrm{~mL}$ of glacial acetic acid $(18 \mathrm{~mol} / \mathrm{L})$ into water and adding either $3 \mathrm{~mL}$ or $5 \mathrm{~mL}$ ammonium hydroxide solution $(13 \mathrm{~mol} / \mathrm{L})$ to make up a buffer with pH 5 or 6 respectively, after dilution to a total volume of $45 \mathrm{~mL}$ in graduated polypropylene vials. $4-\mathrm{ABH}$ user solution $(10 \mathrm{mg} / \mathrm{mL})$ was made by dissolving 250 mg 4-ABH with $25 \mathrm{~mL}$ methanol containing $50 \mathrm{mM} \mathrm{HCl}$.

\subsection{Samples}

A pooled sample of fish feces from Atlantic salmon (Salmo salar) was kindly provided by Skretting AS for method development. In addition, Skretting AS also provided 60 samples of feces from individual fish from a health trial conducted under commercial farming conditions in Southern Norway (production site no. 11971, Store Teistholmen, Rogaland, Grieg Seafood ASA). The fish had an average weight of $240 \mathrm{~g}$ and were anesthetized by an overdose with Finquel (MS-222) before sampling. Feces samples were kept frozen $\left(-18^{\circ} \mathrm{C}\right)$ prior to analysis. Individual fish were sampled a few days after being transferred by well boat from a freshwater facility on land to open sea cages in the fjord. Twenty 
fish were sampled 4 days after transfer, and additional 10 fish were sampled on day 5, 6, 7 , and 8 after transfer.

\subsection{Sample Preparation}

\subsubsection{Fish Feces Supernate}

A pooled sample of fish feces was applied for method development and validation. $2.5 \mathrm{~g}$ of pooled fish feces was transferred to a $10 \mathrm{~mL}$ polypropylene tube with a screw cap (Sarstedt, Nümbrecht, Germany) followed by addition of $7 \mathrm{~mL}$ water, manual shaking for $1 \mathrm{~min}$, and finally centrifugation at $2000 \mathrm{x}$ g for $5 \mathrm{~min}$. Particle-free pooled supernate from multiple such preparations was transferred to large $50 \mathrm{~mL}$ polypropylene tubes (Teknolab, Ski, Norway) and stored frozen until use.

\subsubsection{Deconjugation}

Deconjugation experiments were performed in triplicates with $500 \mu \mathrm{L}$ aliquots of the pooled supernate transferred to $2 \mathrm{~mL}$ polypropylene (PP) microcentrifuge tubes (Teknolab). Both deconjugation and subsequent liquid-liquid extraction (LLE) were carried out in the same vial. For optimization of the enzymatic hydrolysis, $50 \mu \mathrm{L}$ ammonium acetate buffer (2M, pH 5 or 6$)$ was added to the $500 \mu \mathrm{L}$ supernate samples, followed by $20 \mu \mathrm{L}$ of enzyme solution, and finally incubation at $60^{\circ} \mathrm{C}$ for 1 hour. Upon identification of beta-glucuronidase from Helix Pomatia as the most promising enzyme, further optimization was carried out with using 10, 20, 30, 40 and $50 \mu \mathrm{L}$ enzyme solution, and also by incubation at $60{ }^{\circ} \mathrm{C}$ for $1,2,3$, and 24 hours. Chemical hydrolysis was attempted by adding $20 \mu \mathrm{L}$ of $2,4,6,8$, or $10 \%(\mathrm{v} / \mathrm{v})$ of either sulfuric acid or ammonium hydroxide, followed by incubation at $60^{\circ} \mathrm{C}$ for 1 hour.

After deconjugation, samples were processed by an LLE procedure as follows: 50 $\mu \mathrm{L}$ internal standard user solution, $700 \mu \mathrm{L}$ MTBE, and $100 \mu \mathrm{L}$ saturated $\mathrm{NaCl}$ was added to all vials, followed by manual shaking for $1 \mathrm{~min}$, and centrifugation for $5 \mathrm{~min}$ at $2000 \mathrm{x}$ G. $400 \mu \mathrm{L}$ of the solvent phase was transferred to a new $2 \mathrm{~mL}$ PP tube and evaporated to dryness by vacuum centrifugation at $60{ }^{\circ} \mathrm{C}$ for $20 \mathrm{~min}$ (Eppendorf, Hamburg, Germany). Derivatization was carried out by adding $20 \mu \mathrm{L} 4-\mathrm{ABH}$ user solution, incubating at room temperature for $30 \mathrm{~min}$ before adding $40 \mu \mathrm{L}$ water and transfer to $0.3 \mathrm{~mL}$ PP autosampler vials (VWR). Similar sample volume $(500 \mu \mathrm{L})$ of calibration standard solutions, blank water, and untreated feces supernate were prepared by the same LLE and derivatization procedure and analyzed together in the same series using instrumental analysis by LC-MS/MS. Cortisol concentrations in supernate samples were calculated by internal standard calibration.

\subsubsection{Extraction}

LLE was optimized by the processing of $500 \mu \mathrm{L}$ supernate samples that had been deconjugated by incubation with $50 \mu \mathrm{L}$ of ammonium acetate buffer $(\mathrm{pH} 6)$ and $20 \mu \mathrm{L}$ Helix Pomatia enzyme solution for $60 \mathrm{~min}$ at $60^{\circ} \mathrm{C}$. To these samples, $700 \mu \mathrm{L}$ of either a water-immiscible solvent (MTBE, ethyl acetate, or butanol) or a water-miscible solvent (ACN or 1-propanol) was added for performing respectively either LLE or SALLE. The saturated $\mathrm{NaCl}$ solution was then added, either $100 \mu \mathrm{L}$ or $600 \mu \mathrm{L}$ in case of LLE, and 600 $\mu \mathrm{L}$ in case of SALLE. After shaking and centrifugation, $400 \mu \mathrm{L}$ of the top solvent phase was transferred to an empty vial containing $50 \mu \mathrm{L}$ of internal standard user solution, followed by evaporation and derivatization, as described above. All experiments were performed in triplicates and allowed optimization of relative extraction recovery measured by the response factor (analyte peak area / internal standard peak area) and simultaneous assessment of the degree of ion suppression as the reduced peak area of internal standard. Optimization aimed for the highest combined effect of extraction recovery and reduced ion suppression to increase the detectability of cortisol. 


\section{4. $L C-M S / M S$}

Derivatized sample extracts were analyzed by using an Acquity UPLC (Waters, Milford, MA, USA) coupled with a Quattro Premier XE mass spectrometer (Waters). $20 \mu \mathrm{L}$ of sample volume was injected onto a BEH C18 reversed-phase column with $2.1 \mathrm{~mm} \mathrm{ID,}$ $100 \mathrm{~mm}$ length, and $1.7 \AA$ particle size. The mobile phase consisted of A) $0.2 \%(\mathrm{v} / \mathrm{v})$ concentrated ammonium hydroxide in water mixed with B) methanol at a flow rate of 0.25 $\mathrm{mL} / \mathrm{min}$. The linear step gradient was as follows: $0 \mathrm{~min}(1 \% \mathrm{~B}), 3.5 \mathrm{~min}(70 \% \mathrm{~B}), 4-4.5 \mathrm{~min}$ $(95 \% \mathrm{~B}), 4.6-6 \mathrm{~min}(1 \% \mathrm{~B})$.

Hydrazone derivatization products of cortisol (cortisol-4ABH) and D4 labeled cortisol internal standard (D4 cortisol-4ABH) were ionized by positive electrospray ionization (ESI+) using $1 \mathrm{kV}$ capillary voltage and $50 \mathrm{~V}$ cone voltage. Detection was done by multiple reaction monitoring (MRM) using $35 \mathrm{~V}$ collision voltage. MRM transitions were with the following m/z values: $496.25>119.8$ for cortisol- $4 \mathrm{ABH}$ and $500.25>119.8$ for D4 cortisol-4$\mathrm{ABH}$. The hydrazones eluted with a retention time of 4.2 min when using the UPLC conditions described above.

\subsection{Method Validation}

\subsubsection{Final Method}

The method for measurement of total cortisol in fish feces was established as follows:

1. Exact weighing of $0.2-0.5 \mathrm{~g}$ fish feces in a microcentrifuge vial

2. Adding $1000 \mu \mathrm{L}$ water to the vial, followed by 1 min shaking and 5 min centrifugation at $2000 \mathrm{xG}$. Total supernate volume was estimated by adding water and feces volumes together and assuming feces density of $1 \mathrm{~g} / \mathrm{mL}$.

3. Transfer $500 \mu \mathrm{L}$ particle-free supernate to a new vial, then add $50 \mu \mathrm{L}$ ammonium acetate buffer and $20 \mu \mathrm{L}$ enzyme solution, before incubation at $60^{\circ} \mathrm{C}$ for $60 \mathrm{~min}$. Either beta-glucuronidase enzyme from Helix pomatia or from abalone snails (Abalonase ${ }^{\mathrm{TM}}$ ) was applied in the final method.

4. LLE procedure was performed by adding $50 \mu \mathrm{L}$ internal standard user solution, $100 \mu \mathrm{L}$ saturated $\mathrm{NaCl}$ solution and $700 \mu \mathrm{L}$ MTBE to the vial. This step was also done with $500 \mu \mathrm{L}$ of calibration standard solutions, blanks, and quality control sample (pooled feces supernate sample).

5. Shaking the vial for $1 \mathrm{~min}$, followed by centrifugation at $2000 \times \mathrm{xG}$ for $5 \mathrm{~min}$.

6. $400 \mu \mathrm{L}$ of top solvent phase was transferred to a new vial, followed by evaporation to dryness in a vacuum centrifuge at $60^{\circ} \mathrm{C}$ for $20 \mathrm{~min}$.

7. $20 \mu \mathrm{L}$ of derivatization reagent solution was added to the vial, followed by $30 \mathrm{~min}$ incubation at room temperature, and finally $40 \mu \mathrm{L}$ water was added.

8. The derivatized sample extract was transferred to an autosampler vial with microvolume insert $(0.3 \mathrm{~mL})$ for LC-MS/MS analysis and quantification of cortisol in feces supernate by internal standard calibration.

9. The concentration of cortisol in fish feces was reported in w/w units of ng/g (cortisol/feces) and was calculated as follows:

$\underline{\text { [concentration in feces supernate] } \times \text { [total supernate volume] }}$ [weight of feces]

\subsubsection{Method Validation Procedure}

Repeatability (intra series precision) and reproducibility (intra series precision) were investigated by analyzing 10 replicates of the pooled feces supernate the same day and between days, and with a new calibration standard curve prepared each day. Limit of detection (LOD) and limit of quantification (LOQ) were investigated by analyzing 10 
replicates of a surrogate blank sample (water) and by calculating concentrations at response factors made up of average blank plus either 3 times the standard deviation (SD) or $10 \times$ SD of the blank for estimating the LOD and LOQ respectively. Linearity was assessed from the calibration curve of calibration standard solutions extracted together with samples. Accuracy was assessed by analyzing pooled feces supernate, both unspiked and spiked to three concentration levels with known amounts of cortisol.

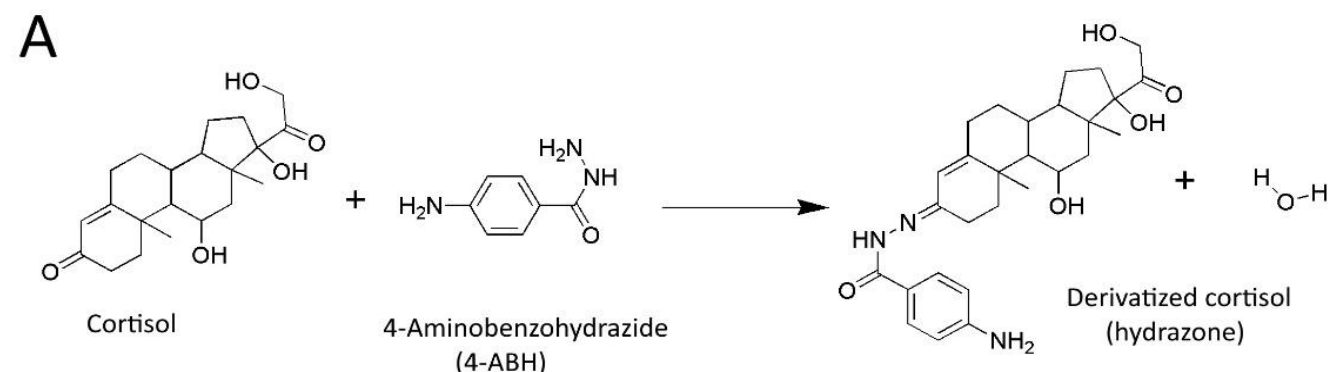

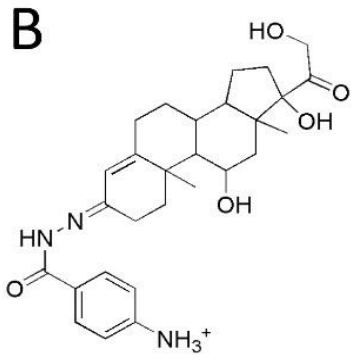

Protonated molecular ion $(\mathrm{m} / \mathrm{z}$ 496.25)

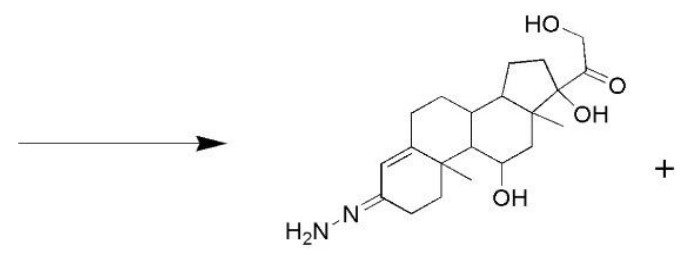

Neutral loss

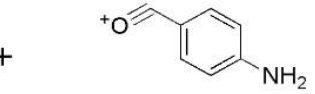

Fragment ion (m/z 119.8)

Figure 1. Molecular structures for illustration of (A) derivatization reaction between cortisol and 4$\mathrm{ABH}$ to the hydrazone and (B) collision-induced dissociation (CID) reaction taking place in the collision cell of the mass spectrometer for fragmentation of the protonated molecular ion of derivatized cortisol and formation of the oxonium fragment ion.

\section{Results}

\subsection{Method Development}

\subsubsection{Instrumental Analysis}

Cortisol and D4 cortisol reacted with $4-\mathrm{ABH}$ in methanol at room temperature to produce a high yield of hydrazones after $30 \mathrm{~min}$ (Figure 1A). Peak splitting was observed in the chromatogram when injecting $20 \mu \mathrm{L}$ of derivatized cortisol in pure methanol, while dilution with water to $33 \%$ methanol resulted in a sharp peak. About 4.5 times higher peak height was observed for derivatized cortisol when using $0.2 \%$ ammonium hydroxide $(\mathrm{pH} 11)$ in the mobile phase instead of the more conventional $0.2 \%$ formic acid ( $\mathrm{pH}$ 2.5). The high $\mathrm{pH}$ mobile phase was compatible with the BEH C18 stationary phase and was applied in further work towards the final method. Protonated molecular ion adduct of derivatized cortisol was the dominating signal in the mass spectrum and was therefore applied for MRM detection. The observed fragment ions of protonated hydrazones of both cortisol and D4 cortisol had m/z 119.8 and were assigned to the common oxonium ion derived from the reagent part of the hydrazone molecules (Figure 1B). With optimized conditions, the peak height of derivatized cortisol was 10 times higher than the peak height of the same amount of underivatized cortisol. 
Chemical hydrolysis with both acid $\left(\mathrm{H}_{2} \mathrm{SO}_{4}\right)$ and base $\left(\mathrm{NH}_{3}\right)$ all resulted in lower measured cortisol concentrations than untreated feces supernate (Figure 2A). Deconjugation with beta-glucuronidase from Helix pomatia clearly released more cortisol from feces supernate than three other enzyme solutions tested (Figure 2A) and was more efficient at pH 6 vs pH 5 (Figure 2B). Adding $20 \mu \mathrm{L}$ of this enzyme solution was found to be sufficient, and an increase above this volume was not found to release significantly higher amounts of cortisol (Figure 2C). Similarly, incubation at $60{ }^{\circ} \mathrm{C}$ for more than $1 \mathrm{~h}$ did not produce significantly higher concentrations of cortisol in the fish feces supernate samples (Figure 2D).

\section{A}

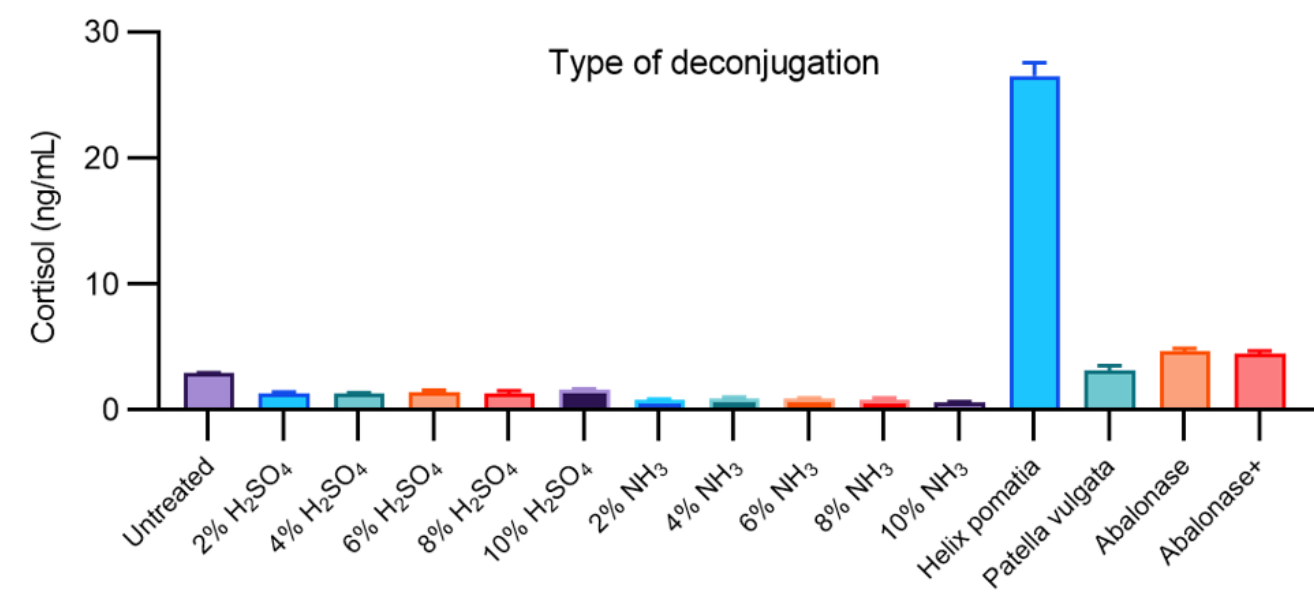

B

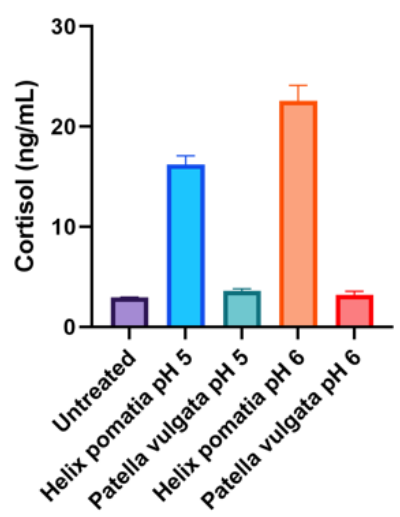

C

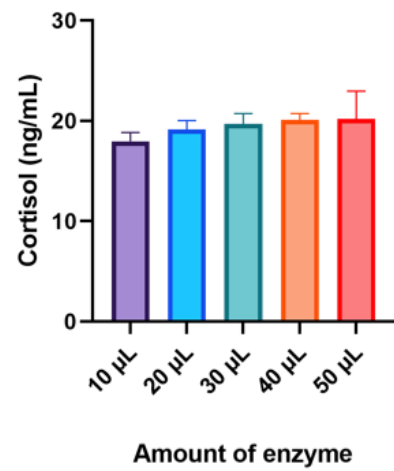

D

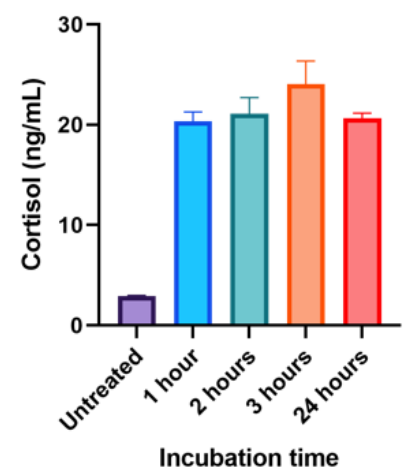

Figure 2. Deconjugation experiments showing various amounts of cortisol released from the fish feces supernate, measured as mean cortisol concentrations with $1 \times \mathrm{SD}$ error bars. (A) Acidic $\left(\mathrm{H}_{2} \mathrm{SO}_{4}\right)$ and basic $\left(\mathrm{NH}_{3}\right)$ hydrolysis compared with enzymatic deconjugation using four different beta-glucuronidases, (B) deconjugation with two different enzyme solutions tested at pH 5 and 6, (C) deconjugation at $\mathrm{pH} 6$ with different volumes of Helix pomatia enzyme solution added, and (D) deconjugation at $\mathrm{pH} 6$ with $20 \mu \mathrm{L}$ of Helix pomatia enzyme solution added and different times of incubation at $60^{\circ} \mathrm{C}$.

\subsubsection{Optimization of Extraction}

In the salting-out assisted liquid-liquid extraction (SALLE) experiments, phase separation was achieved for acetonitrile and propanol when adding as much as $600 \mu \mathrm{L}$ of saturated $\mathrm{NaCl}$ to the supernatants. SALLE with acetonitrile resulted in the highest relative recovery of cortisol (Figure 3A). However, SALLE with either acetonitrile or propanol both resulted in severe ion suppression for cortisol, caused by co-extracted matrix components (Figure 3B). In the liquid-liquid extraction (LLE) experiments, comparable 
extraction recoveries were observed for MTBE, butanol, and ethyl acetate. For MTBE, a small but significant increase in recovery was observed when adding more salt $(600 \mu \mathrm{L}$ vs $100 \mu \mathrm{L}$ of saturated $\mathrm{NaCl}$ ). When extracting with MTBE, however, the addition of more salt also resulted in largely more ion suppression (Figure 3B). By assessing the combined effect of recovery and ion suppression, the highest overall detectability of cortisol was achieved by LLE with MTBE and with $100 \mu \mathrm{L}$ of saturated $\mathrm{NaCl}$ added.

A

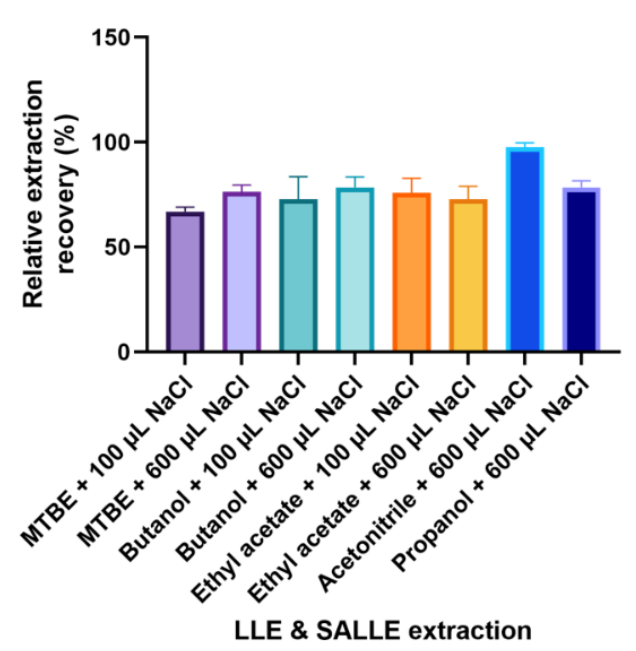

B

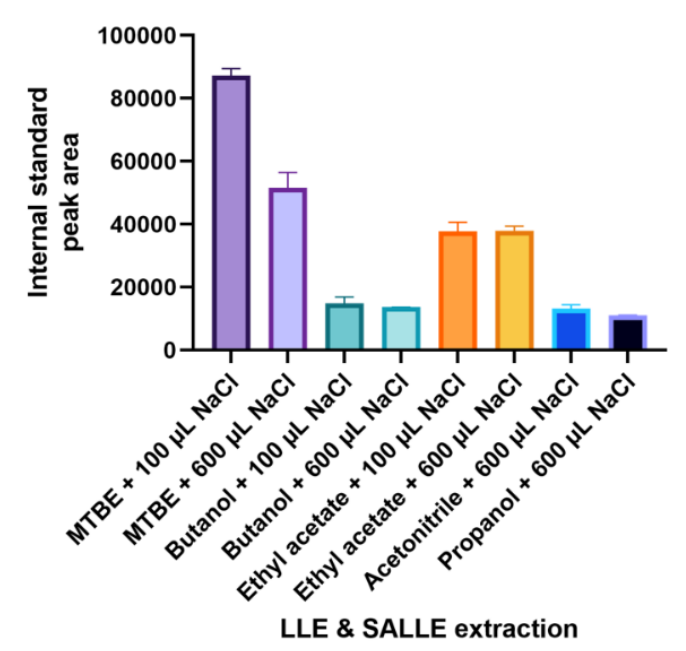

Figure 3. Liquid-liquid extraction (LLE) and salting-out assisted liquid-liquid extraction (SALLE) experiments showing (A) relative extraction recoveries with different solvents and salt added and (B) peak area of the internal standard added after extraction to assess the degree of ion suppression caused by co-extracted matrix components.

\subsection{Method Validation}

Method LOD of $0.04 \mathrm{ng} / \mathrm{mL}$ and LOQ of $0.09 \mathrm{ng} / \mathrm{mL}$ were estimated for part of the method involving analysis of $500 \mu \mathrm{L}$ supernate samples. By considering a 3.8 times dilution of wet feces with water, this corresponded to a total method LOD of $0.15 \mathrm{ng} / \mathrm{g}$ and LOQ of $0.34 \mathrm{ng} / \mathrm{g}$ in the original samples. By later inspection of the signal-to-noise (S/N) ratio in chromatograms from analysis of individual fish feces deconjugated with Abalonase $^{\mathrm{TM}}$ enzyme and found to contain cortisol around $1 \mathrm{ng} / \mathrm{g}$ did, in fact confirm such a low LOQ. The calibration curve was found to be linear $\left(\mathrm{R}^{2}>0.997\right)$ up to $100 \mathrm{ng} / \mathrm{mL}$, corresponding to a concentration in fish feces of $380 \mathrm{ng} / \mathrm{g}$. Accuracy was investigated by spiking cortisol into aliquots of pooled feces supernate, which resulted in the following sample concentration increases: 4.8, 9.6, and $19 \mathrm{ng} / \mathrm{mL}$. By analyzing both spiked and unspiked samples, the recovery estimated at these three levels were 114, 126, and 127\%. By analyzing the very homogenous pooled feces supernate, both intra series and intermediate precision were with a CV of $11 \%$. However, by analyzing individually weighed fish feces samples according to the final method protocol, a somewhat higher but still acceptable CV of $17 \%$ was estimated.

\subsection{Application of the Method}

The novel method was applied for analysis of feces collected from individual fish (Atlantic salmon) a few days after transfer to seawater. For this work, we applied the highly characterized commercial enzyme from Abalone snails (Abalonase ${ }^{\mathrm{TM}}$ ) which was a pure beta-glucuronidase. High levels of cortisol, $437 \pm 293 \mathrm{ng} / \mathrm{g}$, were found four days after transfer but the levels gradually decreased to $74 \pm 55 \mathrm{ng} / \mathrm{g}$ at day eight after transfer (Figure 4). 


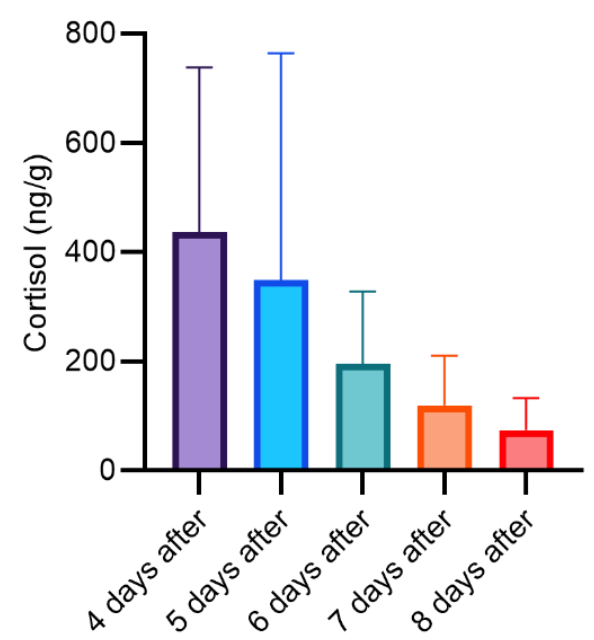

Figure 4. Concentrations of cortisol measured in samples of feces from individual fish transferred from freshwater to seawater.

\section{Discussion}

Because of the rather low concentrations of cortisol in fish feces, it soon became evident that an improved detectability was needed. This was partially achieved by the derivatization with 4-ABH and optimization of LC-MS/MS conditions, especially by using high injection volume and ammonium hydroxide in the mobile phase. When we previously investigated several derivatization reagents for improved detectability of steroid hormones in saliva, the hydrazones formed with 4-ABH were found to produce the highest increase in signal response for cortisol, and again four times higher when using ammonium hydroxide in the mobile phase [9]. However, it was also discovered that many of the hydrazone derivatization products exhibited chromatographic peak splitting, which was the case also for cortisol. This phenomenon was not fully understood at the time and led to some erroneous speculation over possible isomerization. In the present work, we discovered that a dilution of samples with water, after derivatization, reduced the sample solvent elution strength and resulted in the elimination of the peak splitting. The final sample solvent volume was kept low $(60 \mu \mathrm{L})$ and contained only $33 \%$ methanol added from the derivatization solution.

An additional contribution to improved detectability was the use of an optimized LLE in the sample preparation. However, fish feces contain many polar matrix components that will be co-extracted in various amounts together with cortisol. Indeed, increased ion suppression was observed when using polar solvents such as butanol, acetonitrile, and propanol for the extraction. Fortunately, the rather non-polar MTBE was found to extract a satisfactory amount of cortisol from the supernate. Interestingly, attempts to increase extraction recovery by adding more salt also produced more ion suppression. This was explained by the fact that more salt makes the aqueous phase even more polar and will thereby transfer more matrix components to the solvent phase.

Deconjugation of cortisol phase II metabolites was best achieved by using beta-glucuronidase from Helix pomatia. The other three enzymes all resulted in the sub-optimal release of cortisol from the feces supernate samples. The most straightforward explanation for this is that cortisol is mainly conjugated with sulfate, and the fact that the solution from Helix pomatia contains some amount of sulfatase; $<7500 \mathrm{U} / \mathrm{mL}$ according to specifications. The activity of the contained sulfatases could be especially efficient for deconjugation of cortisol, hence explaining the large difference between this enzyme solution and a 
commercial product with a more controlled, although slightly lower, sulfatase activity (Abalonase $\mathrm{TM}^{\mathrm{TM}}$ ). For investigation of stress in individual fish, we finally decided to apply the highly characterized pure beta-glucuronidase from the abalone snail (Abalonase ${ }^{\mathrm{TM}}$ ). Although this enzyme was only the second most efficient for deconjugation, it was still believed to offer a more consistent release of cortisol. It was assumed to be less affected by lot-to-lot variations, which are more likely encountered with the enzyme solution from Helix pomatia that was a crude gastric juice solution from the snails.

This work is the first to present a method for quantification of either free or conjugated total cortisol in fish feces by LC-MS/MS. Samples from individual fish were analyzed and thereby demonstrating the method as a new tool for monitoring stress in farmed fish. In fish recently transferred from freshwater to seawater, the level of stress is known to be high [10]. Indeed, our new method was able to detect significant stress in fish on day four after transfer to sea. Moreover, the mean cortisol level decreased steadily from above $400 \mathrm{ng} / \mathrm{g}$ to below $100 \mathrm{ng} / \mathrm{g}$ on day eight after transfer to seawater. Hence, the method shows great potential for future monitoring of fish welfare. More work is needed to establish reference values, but preliminary data indicate that the lower levels of cortisol found on day eight after transfer were still significantly elevated compared to levels found in unstressed fish.

A previous study with parrotfishes [6], where fecal cortisol was quantified by HPLC$\mathrm{UV}$, found a baseline cortisol concentration of $3.4 \pm 1.1 \mathrm{ng} / \mathrm{g}$, increasing to $36.9 \pm 7.3 \mathrm{ng} / \mathrm{g}$ in stressed fish. These levels are in line with our findings although our data reveals that the sum of free and conjugated cortisol in feces can reach levels of more than $1000 \mathrm{ng} / \mathrm{g}$ in feces from Atlantic salmon under stressful conditions. Future work may involve testing other enzymes for consistent deconjugation of both glucuronidated and sulfated cortisol. It will also be necessary to fully explore the biomarker potential of the method by analyzing samples collected from fish after other stressful events, for instance during an outbreak of disease.

\section{Supplementary Materials: None.}

\section{Author Contributions:}

Conceptualization, David Knudsen and Cato Brede; Data curation, Vanessa Meling, David Knudsen, Per Ola Rønning and Cato Brede; Formal analysis, Vanessa Meling, Kjetil Berge, Per Ola Rønning and Cato Brede; Investigation, Vanessa Meling, Kjetil Berge, David Knudsen and Cato Brede; Methodology, Vanessa Meling, Kjetil Berge, David Knudsen, Per Ola Rønning and Cato Brede; Project administration, David Knudsen; Resources, Kjetil Berge, David Knudsen and Cato Brede; Supervision, Per Ola Rønning and Cato Brede; Validation, Vanessa Meling, Kjetil Berge, David Knudsen, Per Ola Rønning and Cato Brede; Visualization, Vanessa Meling, David Knudsen and Cato Brede; Writing - original draft, Vanessa Meling and Cato Brede; Writing - review \& editing, Vanessa Meling, Kjetil Berge, David Knudsen, Per Ola Rønning and Cato Brede. All authors have read and agreed to the published version of the manuscript.

Funding: This research received no external funding.

Institutional Review Board Statement: Ethical review and approval were waived for this study due to sample collection performed during routine inspection of the farmed fish for other purposes.

Informed Consent Statement: Not applicable.

Data Availability Statement: Not applicable.

Acknowledgments: The authors would like to thank Trude A. Sandvik and Asgeir Husa at Dr. Fürst Medisinsk Laboratorium AS, as well as Christian Thorstensen at Oslo University Hospital, for their kind donations of instruments.

Conflicts of Interest: The authors declare no conflict of interest.

Sample Availability: Not applicable. 


\section{References}

1. Johansen, U.; Bull-Berg, H.; Vik, L.H.; Stokka, A.M.; Richardsen, R.; Winther, U. The Norwegian Seafood Industry - Importance for the National Economy. Marine Policy 2019, 110, 103561, doi:10.1016/j.marpol.2019.103561.

2. Stien, L.H.; Tørud, B.; Gismervik, K.; Lien, M.E.; Medaas, C.; Osmundsen, T.; Kristiansen, T.S.; Størkersen, K.V. Governing the Welfare of Norwegian Farmed Salmon: Three Conflict Cases. Marine Policy 2020, 117, 103969, doi:10.1016/j.marpol.2020.103969.

3. Sadoul, B.; Geffroy, B. Measuring Cortisol, the Major Stress Hormone in Fishes. Journal of Fish Biology 2019, 94, 540-555, doi:10.1111/jfb.13904.

4. Cook, N. Review: Minimally Invasive Sampling Media and the Measurement of Corticosteroids as Biomarkers of Stress in Animals. Canadian Journal of Animal Science 2012, 92, 227-259, doi:10.4141/cjas2012-045.

5. Cao, Y.; Tveten, A.-K.; Stene, A. Establishment of a Non-Invasive Method for Stress Evaluation in Farmed Salmon Based on Direct Fecal Corticoid Metabolites Measurement. Fish E Shellfish Immunology 2017, 66, 317-324, doi:10.1016/j.fsi.2017.04.012.

6. Turner, J.W.; Nemeth, R.; Rogers, C. Measurement of Fecal Glucocorticoids in Parrotfishes to Assess Stress. General and Comparative Endocrinology 2003, 133, 341-352, doi:10.1016/S0016-6480(03)00196-5.

7. Keevil, B.G. LC-MS/MS Analysis of Steroids in the Clinical Laboratory. Clinical Biochemistry 2016, 49, 989-997, doi:10.1016/j.clinbiochem.2016.04.009.

8. Marcos, J.; Pozo, O.J. Derivatization of Steroids in Biological Samples for GC-MS and LC-MS Analyses. Bioanalysis 2015, 7, 25152536, doi:10.4155/bio.15.176.

9. Nadarajah, N.; Skadberg, Ø.; Adaway, J.; Brede, C. Multiplexed Analysis of Steroid Hormones in Saliva by LC-MS/MS with 2Hydrazinopyridine Derivatization. Clinical Mass Spectrometry 2017, 4-5, 1-10, doi:10.1016/j.clinms.2017.08.001.

10. McCormick, S.D.; Regish, A.M.; Christensen, A.K.; Björnsson, B.T. Differential Regulation of Sodium-Potassium Pump Isoforms during Smolt Development and Seawater Exposure of Atlantic Salmon. Journal of Experimental Biology 2013, 216, 1142-1151, doi:10.1242/jeb.080440. 